\title{
Stability of the liquid markers chromium (III) and cobalt (III)-EDTA in autoclaved, clarified rumen fluid
}

\author{
Mary Beth Hall ${ }^{1 *}$ and Peter J. Van Soest ${ }^{2}$ \\ ${ }^{1}$ US Dairy Forage Research Center, USDA-Agricultural Research Service, Madison, WI 53706 \\ ${ }^{2}$ Professor Emeritus, Department of Animal Science, Cornell University, Ithaca, NY 14853
}

\begin{abstract}
An ideal digesta marker for use in feeding studies is inert, unabsorbable, and moves with the portion of the digesta it is intended to mark. Both chromium (III) and cobalt (III) salts of EDTA (CrEDTA and CoEDTA, respectively) have been used as digesta liquid markers in studies with dairy cattle. Although a small portion of these markers is known to be absorbed and excreted in urine, the markers are assumed to remain ionically bound in the digesta. The degree to which these salts remain bound in solution can be determined through spectrophotometric measurement at the wavelength $(\lambda)$ of peak absorbance of these colored compounds. The objective of this study was to evaluate whether CrEDTA and CoEDTA dissociate during incubation in autoclaved, clarified rumen fluid (ACRF), as indicated by changes in absorbance. In a complete block design with separate replicated analytical runs, approximately $40 \mathrm{mg} / \mathrm{L}$ of $\mathrm{Cr}$ from CrEDTA or Co from CoEDTA were incubated in 2 separate preparations of ACRF from 2 lactating Holstein cows, in water (CrEDTA), or in MES buffer (CoEDTA), and appropriate reagent blanks. Solution $\mathrm{pH}$ were approximately 6.0. Samples were incubated for $24 \mathrm{~h}$ at $39^{\circ} \mathrm{C}$ with absorbance measurements recorded at $0,1,2,4,6,22$, and 24 . The CrEDTA was measured at $\lambda=541 \mathrm{~nm}$, CoEDTA at $\lambda$ $=535 \mathrm{~nm}$, and both were measured with wavescans of $\lambda$ $=330$ to $700 \mathrm{~nm}$. At their peak $\lambda$, both CrEDTA in water and CoEDTA in MES buffer maintained absorbance values throughout the incubation, whereas, in ACRF, CrEDTA absorbance decreased by $9 \%$ at $0 \mathrm{~h}$, and by up to $14 \%$ by $24 \mathrm{~h}$; CoEDTA showed a gradual decline over time, with approximately $4 \%$ loss in absorbance by $24 \mathrm{~h}$. Responses differed by ACRF preparation. Both markers in ACRF showed increases and decreases over time in absorbance at $\lambda=330$ and $380 \mathrm{~nm}$, though the
\end{abstract}

Received September 28, 2018.

Accepted April 9, 2019.

*Corresponding author: marybeth.hall@ars.usda.gov changes were more marked for CrEDTA; markers not in ACRF showed no change in absorbance at these $\lambda$. Changes in the absorbance values at $\lambda=330$ and 380 nm suggest that soluble phenolic compounds may be involved in the exchange of metals with EDTA, but that does not preclude involvement of other components in rumen fluid. Both CrEDTA and CoEDTA incubated in ACRF showed declines over time in absorbance at their peak $\lambda$, suggesting that the metals dissociated from EDTA. The apparent dissociation indicates that these liquid markers are not inert as had been assumed. Key words: liquid, marker, passage, rumen

\section{INTRODUCTION}

Liquid markers for digesta to estimate liquid passage, rumen volume, and ruminal substrate disappearance have been used in studies with lactating dairy cattle (Weisbjerg et al., 1998; Shingfield et al., 2008). An ideal digesta marker for use in feeding studies is indigestible, unabsorbable, moves with the portion of the digesta it is intended to mark (Faichney, 1993), and should be accurately and specifically measurable. However, all markers have limitations. The longest used liquid marker is the organic polymer polyethylene glycol (PEG), typically of a molecular weight of 4,000 or greater. Although PEG may complex with feeds containing tannins (Teeter and Owens, 1983), it has been reported to not (Teeter and Owens, 1983) or minimally (Downes and McDonald, 1964) be recovered in urine, and has not been found to be appreciably degraded in the gut (Teeter and Owens, 1983). A primary criticism of PEG is the need to measure it by a turbidimetric assay, which is affected by technique (Downes and McDonald, 1964).

The EDTA salts of chromium (III; CrEDTA) and cobalt (III; CoEDTA) were developed as liquid markers to allow greater specificity of analysis, with CoEDTA introduced as a non-Cr-containing material to facilitate use of multiple markers (Udén et al., 1980). The Cr or Co in these markers can be analyzed specifically using atomic absorption spectroscopy or inductively coupled 
plasma atomic emission spectroscopy (ICP; AOAC Official Method 990.08, AOAC International, 2005). Small portions of both markers are excreted in urine. In lactating dairy cows, CrEDTA and CoEDTA gave urinary excretions of $\mathrm{Cr}$ or Co of approximately $2.4 \%$ of the ruminally infused dose (Shingfield et al., 2008), and a value of $4.7 \%$ of $\mathrm{Cr}$ from ruminally infused CrEDTA was found for sheep (Downes and McDonald, 1964). The degree to which the EDTA salts were absorbed from the rumen was investigated by Dobson et al. (1976). Absorption rates of $\left.{ }^{51} \mathrm{Cr}\right]$ EDTA from the ventral sac of nonlactating dairy cows was small $(0.2 \mathrm{~mL} /$ min) when administered solutions were isotonic or hypotonic relative to plasma, but reached rates of $8 \mathrm{~mL} /$ min in hypertonic solutions. They noted that the high rates of absorption from the rumen were reached within the range of osmotic pressures normally encountered postprandially. In that study, eluate from urine applied to an anion exchange column had a "faint purple color" with a peak absorbance of 542; they concluded that the CrEDTA "passed from the rumen into the blood without appreciable degradation." No similar absorption studies were found for CoEDTA which, like CrEDTA, is a monovalent anion.

An untested assumption regarding CoEDTA and CrEDTA is that these ionically bonded molecules do not dissociate in digesta. Though not explicitly stated, the stability and nonreactivity of CrEDTA was presented in early papers as a point supporting use of such markers (Downes and McDonald, 1964; Binnerts et al., 1968). The assumption of stability is the basis for using the metal-EDTA markers rather than dosing the Co or Cr compounds used to prepare them. The work of Shingfield et al. (2008) has suggested that CoEDTA may dissociate when infused ruminally in dairy cows, resulting in changes in the profile of fatty acids in milk.

A challenge to addressing the question of marker dissociation is the issue of measuring dissociation of a salt in a complex matrix such as ruminal digesta. In such a matrix, it is typically not feasible to measure a compound as an ionically bound salt. For example, we can measure $\mathrm{Na}$ and $\mathrm{Cl}$, but cannot ensure that $\mathrm{Na}$ and $\mathrm{Cl}$ are associated as $\mathrm{NaCl}$. However, because CrEDTA and CoEDTA are colored compounds with distinct absorbance patterns across wavelengths, and have absorbance maxima at specific wavelengths, they can be detected spectrophotometrically (Hamm, 1953; Paraneeiswaran et al., 2015). An example of spectrophotometry being used to assess presence of metal-EDTA markers was the measurement of absorbance at the peak wavelength of CrEDTA to monitor progression of its formation in kinetic studies (Hedrick, 1965). Accordingly, measurement of the absorbance of light across wavelengths of visible light and at specific wavelengths can be used to evaluate whether CrEDTA and CoEDTA are present in their ionically bound, colored forms, or if they have dissociated. The wavelengths of maximum absorption can be slightly affected by $\mathrm{pH}$ with maximum absorption for CrEDTA occurring at $541 \mathrm{~nm}$ at slightly acidic $\mathrm{pH}$ (Cerar, 2015).

The objective of this study was to determine whether CrEDTA and CoEDTA salts dissociate in autoclaved clarified rumen fluid (ACRF). The use of ACRF rather than rumen fluid with microbes removed the effect of microbial action and the complications associated with how their growth, death, and products would alter absorbance, unrelated to changes due to the markers. This approach allowed us to focus the experiment on the effects of compounds present in rumen fluid, and provided solutions that were largely free of particulate matter and microbes that would interfere with absorbance measurements.

\section{MATERIALS AND METHODS}

Autoclaved clarified rumen fluid was prepared using ruminal inoculum obtained from 2 lactating Holstein cows under protocols approved by the Institutional Animal Care and Use Committee of the University of Wisconsin College of Agriculture and Life Sciences. Cows were offered a TMR containing, on a DM basis, $27 \%$ corn silage, $21 \%$ alfalfa silage, $6 \%$ whole linted cottonseed, and $46 \%$ grain mix. The grain mix contained, on a DM basis, $43 \%$ ground corn grain, $12.3 \%$ distillers dried grains and solubles, $16.4 \%$ canola meal, $12.0 \%$ heat-treated soy product, $8.1 \%$ soybean hulls, $2.1 \%$ sodium bicarbonate, $0.8 \%$ added fat, $0.5 \%$ urea, and $4.4 \%$ vitamin and mineral mix. Diets were formulated to meet animal requirements (NRC, 2001). The inoculum from each cow was processed separately to provide 2 sources of ACRF. For each cow, rumen digesta was obtained via the rumen cannula and placed into a warmed, insulated container. The digesta was filtered and squeezed through 4 layers of grade 90 cheesecloth, and filtered through 4 additional layers into an Erlenmeyer flask. The rumen fluid was centrifuged twice at $13,000 \times g$ at $5^{\circ} \mathrm{C}$ for $45 \mathrm{~min}$ in $250-\mathrm{mL}$ bottles, with the supernate decanted into another bottle for the second centrifugation. The $\mathrm{pH}$ of the rumen fluid was adjusted to 6.0 using $1 \mathrm{M} \mathrm{NaOH}$, then autoclaved in sealed serum vials for $30 \mathrm{~min}$. Separate sets of ACRF were prepared for CrEDTA and CoEDTA incubations, with a given set used for the replicate incubations within marker type within 5 to $8 \mathrm{~d}$ of preparation. The day of the incubations with markers, serum vials were opened, contents transferred to $50-\mathrm{mL}$ centrifuge bottles and centrifuged at $13,000 \times g$ for $10 \mathrm{~min}$ at ambient temperature; ACRF from each cow was repooled after centrifugation. 


\section{Liquid Markers}

The lithium salt of CrEDTA was prepared using $\mathrm{Cr}$ (III) acetate hydroxide (Hall and Van Soest, 2019), and that of CoEDTA prepared according to the method of Udén et al. (1980). Solutions of CrEDTA (0.206 g/100 $\mathrm{mL}$ with water) and CoEDTA $(0.185 \mathrm{~g} / 100 \mathrm{~mL}$ of MES buffer; MES) were prepared. Marker concentrations were set so that when mixed with ACRF, they provided a concentration equivalent to $2 \mathrm{~g}$ of $\mathrm{Cr}$ or $\mathrm{Co}$ in $50 \mathrm{~L}$ of rumen liquid; a dose and dilution similar to what would be used in in vivo studies with cows. Two separate replicate solutions were prepared for each marker.

\section{Marker and ACRF Incubations}

Two incubation runs were performed under aerobic conditions for each marker, with CoEDTA and CrEDTA evaluated in separate replicated runs. In each run, the marker solutions, and the diluents $\mathrm{ACRF}$, water, and MES were brought to $39^{\circ} \mathrm{C}$ in a water bath and the $\mathrm{pH}$ adjusted to 6.0 using lactic acid (\#41296-5000, Acros Organics, Thermo Fisher Scientific, Pittsburgh, $\mathrm{PA})$ or $\mathrm{NaOH}(\# 221465,97+\%$, A.C.S. reagent, SigmaAldrich, St. Louis, MO) if needed. The ACRF from each cow was pipetted with a positive displacement pipette (Eppendorf Repeater M4, Eppendorf North America, Hauppauge, NY) to deliver $28 \mathrm{~mL}$ per $50-\mathrm{mL}$ conical tube. Six tubes with $28 \mathrm{~mL}$ of water for CrEDTA runs or with MES for CoEDTA runs were pipetted. To each tube was added $4 \mathrm{~mL}$ of water, MES, or marker solutions pipetted with a positive displacement pipette to produce sample solutions. Tubes were then capped and inverted to mix. Solutions containing ACRF and markers were run singly, and reagent blanks and markers with water or MES were run in duplicate (Table 1).

Each solution was pipetted $(200 \mu \mathrm{L})$ in duplicate into wells of a 96 -well plate using a positive displace- ment repeat pipetter, giving an approximately $5 \mathrm{~mm}$ path length. Once all solutions were pipetted, the plate was placed in a $39^{\circ} \mathrm{C}$ incubating, shaking plate reader (EONC, Biotek Instruments Inc., Winooski, VT), and the remainder of the solutions were capped and placed in the $39^{\circ} \mathrm{C}$ water bath. The solutions in the plate were scanned at $0,1,2,4,6,22$, and $24 \mathrm{~h}$ of incubation at wavelengths of 330 to $700 \mathrm{~nm}$ in increments of 10 $\mathrm{nm}$. At each hour point, the average absorbances of the appropriate reagent blanks were subtracted from the values for the solutions containing CrEDTA or CoEDTA. Values for duplicate samples in the plate reader were averaged for statistical analysis. Wavelength scan data were used to evaluate responses at 380 and 330 $\mathrm{nm}$, and whether markers incubated in water or MES maintained their maximum absorbance at their specific peak wavelength.

The remaining solutions in conical tubes were maintained in a water bath at $39^{\circ} \mathrm{C}$ and used to evaluate changes at peak absorbance over time. The solutions were vortexed, $\mathrm{pH}$ was measured, and a sample removed to measure absorbance at $0,1,2,4,6,22$, and $24 \mathrm{~h}$ of incubation. Absorbance was measured at the peak wavelength for each marker: $541 \mathrm{~nm}$ for CrEDTA solutions and $535 \mathrm{~nm}$ for CoEDTA solutions using cuvettes with a $1 \mathrm{~cm}$ path length in a spectrophotometer (Genesys 10 Vis, \#335900, Thermo Fisher Scientific, Waltham, MA). At the end of each run, remaining solutions were transferred to scintillation vials and frozen at $-20^{\circ} \mathrm{C}$ for future analysis. At each hour point, the average of the appropriate reagent blanks was subtracted from the values for the samples with marker added.

\section{Sample Analyses}

The ACRF-water and ACRF-MES solutions as well as those containing only water or MES were thawed

Table 1. Contents of and numbers of tubes incubated in each analytical run to evaluate dissociation of markers ${ }^{1,2}$

\begin{tabular}{|c|c|c|c|c|c|c|}
\hline \multirow[b]{2}{*}{ Item } & \multicolumn{3}{|c|}{ CrEDTA } & \multicolumn{3}{|c|}{ CoEDTA } \\
\hline & ACRF1 & ACRF2 & Water & ACRF3 & ACRF4 & MES buffer \\
\hline Water & 2 & 2 & 2 & - & - & - \\
\hline CrEDTAa & 1 & 1 & 2 & - & - & - \\
\hline CrEDTAb & 1 & 1 & 2 & - & - & - \\
\hline MES buffer & - & - & - & 2 & 2 & 2 \\
\hline CoEDTAa & - & - & - & 1 & 1 & 2 \\
\hline CoEDTAb & - & - & - & 1 & 1 & 2 \\
\hline
\end{tabular}

${ }^{1}$ Six tubes with $28 \mathrm{~mL}$ of water for chromium-EDTA (CrEDTA) runs or with MES buffer for cobalt-EDTA (CoEDTA) runs were pipetted. To each tube was added $4 \mathrm{~mL}$ of water, MES buffer, or marker solutions pipetted with a positive displacement pipette to produce sample solutions.

${ }^{2} \mathrm{ACRF}=$ autoclaved, clarified rumen fluid (number indicates batch). CoEDTA and CrEDTA: letter indicates replicate. 
and analyzed for organic acids by HPLC (Weimer et al., 1991) and for minerals by ICP (AOAC Official Method 990.08, AOAC International, 2005; model 5100, Agilent Technologies, Santa Clara, CA). Samples containing markers were analyzed for $\mathrm{Cr}$ and $\mathrm{Co}$ by ICP.

\section{Statistics}

The study was performed as a complete block design with data analyzed as repeated measures in time. For samples incubated in the water bath, absorbance values were analyzed using a mixed model that included the fixed effects of ACRF, marker preparation ( $a$ or $b$ in each run), sampling hour, all 2-way interaction terms, and incubation run as a random variable. For the samples incubated in the plate reader, absorbance at 330 and $380 \mathrm{~nm}$ were evaluated with the same model. The covariance structures tested were compound symmetry, heterogeneous compound symmetry, autoregressive, heterogeneous autoregressive, and ante-dependence. Covariance structures were selected on the basis of which gave the minimum Akaike's information criterion. Accordingly, compound symmetry was used for analyses at the wavelengths of maximum absorption for each marker, heterogeneous autoregressive for $\mathrm{pH}$ evaluations, and heterogeneous compound symmetry for analyses of both markers at 330 and $380 \mathrm{~nm}$. For all analyses, the markers in the 2 ACRF preparations, water, or MES were compared within marker type using the orthogonal contrasts water or MES versus ACRF, and ACRF1 versus ACRF2 or ACRF3 versus ACRF4.

Markers not in ACRF subject to wavelength scans in the plate reader were evaluated to determine whether they maintained their peak absorbance, $541 \mathrm{~nm}$ for CrEDTA and $535 \mathrm{~nm}$ for CoEDTA, throughout the incubation. At each time point, the difference between maximum absorbance detected and that measured at $540 \mathrm{~nm}$ for CrEDTA, and of the absorbance at 530 $\mathrm{nm}$ minus that at $540 \mathrm{~nm}$ for CoEDTA were evaluated by run using $t$-tests to determine if the values differed from zero. Differences in mineral and organic acid concentrations between the $2 \mathrm{ACRF}$ preparations used within CrEDTA and CoEDTA runs were analyzed as the difference in concentrations between the reagent blank ACRF preparations in each run. The differences were analyzed using $t$-tests applied to 2 values determined with replicate marker runs. Data for CrEDTA and CoEDTA are not directly comparable because of their analyses with different ACRF sources. Analyses were performed with SAS (version 9.4, SAS Institute Inc., Cary, NC). Least squares means values are presented with standard errors of the difference (SED) unless otherwise stated.

\section{RESULTS AND DISCUSSION}

Measurements made on samples conserved at the end of the analytical runs indicate that comparable amounts of marker were added, and that reagent blanks contained little of the element specific to the marker. Sample solutions that contained CrEDTA had measured $\mathrm{Cr}$ contents averaging $40.2 \mathrm{mg} / \mathrm{L}$, with a maximum of $41.3 \mathrm{mg} / \mathrm{L}$, a minimum of $39.0 \mathrm{mg} / \mathrm{L}$, and a standard deviation of $0.64 \mathrm{mg} / \mathrm{L}$. Reagent blank solutions that included ACRF or water contained 0.01 to $0.03 \mathrm{mg} / \mathrm{L}$ of $\mathrm{Cr}$. The CoEDTA sample solutions containing marker averaged $40.2 \mathrm{mg} \mathrm{Co} / \mathrm{L}$, with a maximum of 41.6, minimum of 38.9, and a standard deviation of $0.89 \mathrm{mg} / \mathrm{L}$. Reagent blank solutions that included ACRF or MES contained between $0.03 \mathrm{mg}$ of $\mathrm{Co} / \mathrm{L}$ and levels that were below detection limits.

As literal standards for comparison, all markers not prepared with ACRF were evaluated spectrophotometrically with wavelength scans from 330 to 700 $\mathrm{nm}$ to determine whether they maintained maximal absorption close to the expected wavelengths over the course of the incubations. Both CrEDTA and CoEDTA maintained maximum absorbance at their specific wavelengths throughout the $24 \mathrm{~h}$ runs. For CrEDTAwater, the difference between the wavelength of the maximum measured absorbance and $540 \mathrm{~nm}$, the measured wavelength closest to the actual maximum of 541 $\mathrm{nm}$ equaled 0 at all time points; the statistical model would not solve because no variation was present. For CoEDTA with its maximum absorbance at $535 \mathrm{~nm}$, the difference between absorbances measured at 530 and $540 \mathrm{~nm}$ was expected to be 0 , if the maximal absorption actually fell at the midpoint between these wavelengths. The difference between absorbances for $530 \mathrm{~nm}$ minus $540 \mathrm{~nm}$ was very close to but differed from 0 at -0.00054 units of absorbance (standard error of the mean; $\mathrm{SE}=0.000086 ; P<0.01)$ for both runs. It is possible that a wavelength slightly higher than $535 \mathrm{~nm}$ is the wavelength of the actual maximum absorbance for CoEDTA.

Reagent blanks prepared with ACRF from different cows showed differences in organic acid and mineral concentrations (Tables 2 and 3). Fermentation acids were not present at detectable levels in the water or MES solutions, and so are omitted from Table 2. For the CrEDTA runs, all organic acids differed $(P<0.05)$ or tended to differ $(0.05 \leq P<0.10)$ between ACRF preparations, whereas for CoEDTA runs, the branched chain VFA did not differ $(P=0.40)$; in all runs, ACRF2 or ACRF4 had the overall greater concentrations of organic acids. The only minerals that did not differ or tend to differ between ACRF preparations within CoEDTA or CrEDTA runs were $\mathrm{Cu}(P=0.80)$ and $\mathrm{Zn}(P$ 
Table 2. Organic acid concentrations $(\mathrm{m} M)$ of reagent blanks containing autoclaved clarified rumen fluid (ACRF) and water or MES buffer (MES)

\begin{tabular}{|c|c|c|c|c|c|c|c|c|c|}
\hline Sample ${ }^{1}$ & Marker $^{2}$ & Diluent & Run & Lact $^{3}$ & $\mathrm{Ac}^{3}$ & Prop $^{3}$ & $\mathrm{But}^{3}$ & $\mathrm{Val}^{3}$ & BCVFA $^{3}$ \\
\hline ACRF1 & CrEDTA & Water & 10 & 7.2 & 81.7 & 29.4 & 13.3 & 1.8 & 3.1 \\
\hline ACRF2 & CrEDTA & Water & 9 & 3.0 & 100.5 & 31.5 & 16.7 & 1.7 & 2.7 \\
\hline$P$-value & CrEDTA & & & 0.03 & $<0.01$ & 0.02 & $<0.01$ & $<0.01$ & 0.048 \\
\hline SEM & CrEDTA & & & 0.19 & 0.10 & 0.08 & 0.03 & 0 & 0.03 \\
\hline ACRF3 & CoEDTA & MES & 11 & 11.9 & 73.0 & 24.6 & 12.5 & 1.4 & 2.4 \\
\hline ACRF4 & CoEDTA & MES & 12 & ND & 75.6 & 38.1 & 12.0 & 1.9 & 2.6 \\
\hline$P$-value & CoEDTA & & & 0.02 & 0.051 & 0.02 & 0.07 & $<0.01$ & 0.40 \\
\hline SEM & CoEDTA & & & 0.29 & 0.26 & 0.38 & 0.04 & 0.005 & 0.13 \\
\hline
\end{tabular}

${ }^{1} \mathrm{ACRF}$ : number indicates batch.

${ }^{2} \mathrm{CrEDTA}=$ chromium-EDTA; CoEDTA $=$ cobalt-EDTA.

${ }^{3}$ Lact $=$ lactate Ac $=$ acetate Prop $=$ propionate; But $=$ butyrate; Val = valerate; $\mathrm{BCVFA}=$ branched-chain VFA.

${ }^{4}$ Not detected.

$=0.61)$ in $\mathrm{ACRF} 1$ versus $\mathrm{ACRF} 2$, and $\mathrm{S}$ for either pair of runs $(P>0.13)$. The CoEDTA solutions had high $\mathrm{S}$ concentrations due to the addition of MES, which contains S. Overall, ACRF2 and ACRF4 preparations had greater mineral concentrations than ACRF1 or ACRF3. Given the finding that differences in ruminal osmotic pressure relative to plasma can alter absorption of CrEDTA (Dobson et al., 1976), and that both minerals and organic acids contribute to the osmolality of ruminal fluid, the difference in mineral and organic acid concentrations of ACRF obtained from different dairy cows sampled at the same time suggests that cows or cows sampled at different times post-feeding may differ in the ruminal absorption of EDTA markers.

\section{Apparent Dissociation of Markers}

It has been reported that $\mathrm{pH}$ can affect absorbance of CrEDTA (Cerar, 2015). The pH of CrEDTA solutions were affected by diluent and time (diluent $\times$ time $P<0.01$, SED $=0.024$; water vs. ACRF, $P<0.01$; ACRF1 vs. ACRF2, $P=0.92$; Figure 1A). Despite the 0.23 unit decrease in $\mathrm{pH}$, CrEDTA-water maintained its absorbance at $541 \mathrm{~nm}$ within $1.1 \%$ of its $0 \mathrm{~h}$ value, and within 0.0013 units of absorbance (Figure 1B), which was similar in performance to CoEDTA-MES, which maintained a more uniform $\mathrm{pH}$ (Figure 1E). The change in $\mathrm{pH}$ may have been due to the action of CrEDTA as a Lewis base, which would not require

Table 3. Mineral concentrations (mg/L) of reagent blanks containing autoclaved clarified rumen fluid (ACRF) and water (W) or MES buffer $(\mathrm{M})^{1}$

\begin{tabular}{|c|c|c|c|c|c|c|c|c|c|c|c|c|c|}
\hline Sample $^{2}$ & $\mathrm{Metal}^{3}$ & Diluent & Run & $\mathrm{Ca}$ & $\mathrm{Cu}$ & $\mathrm{Fe}$ & $\mathrm{K}$ & $\mathrm{Mg}$ & Mn & $\mathrm{Na}$ & $\mathrm{P}$ & S & $\mathrm{Zn}$ \\
\hline ACRF1 & $\mathrm{Cr}$ & W & 10 & 125 & 0.35 & 0.80 & 1,480 & 259 & 3.56 & 2,907 & 524 & 22.4 & 1.50 \\
\hline ACRF2 & $\mathrm{Cr}$ & W & 9 & 167 & 0.32 & 0.83 & 1,672 & 294 & 3.95 & 3,091 & 572 & 23.3 & 1.45 \\
\hline$P$-value & $\mathrm{Cr}$ & & & $<0.01$ & 0.80 & 0.06 & 0.01 & 0.04 & $<0.01$ & 0.05 & 0.02 & 0.14 & 0.61 \\
\hline SEM & $\mathrm{Cr}$ & & & 0.55 & 0.02 & 0.005 & 3.75 & 2.75 & 0.005 & 18.4 & 1.80 & 0.36 & 0.04 \\
\hline ACRF3 & $\mathrm{Co}$ & M & 11 & 99.1 & 0.28 & 0.45 & 1,307 & 191 & 1.55 & 3,099 & 551 & 272 & 1.21 \\
\hline$P$-value & Co & & & 0.02 & 0.08 & $<0.01$ & 0.01 & 0.01 & 0.02 & 0.04 & 0.07 & 0.67 & 0.05 \\
\hline SEM & $\mathrm{Co}$ & & & 1.80 & 0.005 & 0.00 & 3.55 & 1.60 & 0.06 & 4.70 & 3.15 & 2.76 & 0.01 \\
\hline \multirow[t]{4}{*}{$\mathrm{W}$ or $\mathrm{M}$} & $\mathrm{Cr}$ & W & 9 & 0.98 & 0.22 & 0.04 & 80.8 & 0.14 & 0.01 & 34.2 & 0.30 & 1.38 & 1.59 \\
\hline & $\mathrm{Cr}$ & W & 10 & 0.81 & 0.21 & 0.02 & 76.5 & 0.07 & 0.01 & 33.4 & 0.33 & 0.53 & 1.77 \\
\hline & $\mathrm{Co}$ & M & 11 & 0.94 & 0.12 & $\mathrm{BD}^{4}$ & 63.0 & 0.13 & 0.03 & 734 & 0.30 & 1927 & 0.93 \\
\hline & Co & M & 12 & 0.80 & 0.12 & $\mathrm{BD}$ & 59.5 & 0.10 & 0.02 & 743 & 0.31 & 1939 & 0.91 \\
\hline
\end{tabular}

\footnotetext{
${ }^{1}$ Values are arithmetic means.

${ }^{2} \mathrm{ACRF}$ : number indicates batch.

${ }^{3}$ Metal associated with EDTA.

${ }^{4} \mathrm{BD}=$ below detection limit.
} 
dissociation of the complex. Water rather than buffer was used as the diluent for CrEDTA based on preliminary work that indicated that CrEDTA in water was less susceptible to $\mathrm{pH}$ change than CoEDTA, which required use of a buffer to maintain $\mathrm{pH}$. The CrEDTAACRF showed a 9\% decrease in absorbance relative to CrEDTA-water at the initial reading (Figure 1B). This change occurred within the 12 to 22 min time between pipetting and mixing the CrEDTA solutions and reading their absorbance at $0 \mathrm{~h}$. The CrEDTAACRF absorbances declined further, and then diverged in behavior by ACRF preparation (effect of diluent $x$ time, $P<0.01$, SED 0.0006; water vs. ACRF, $P<$ 0.01; ACRF1 vs. ACRF2, $P<0.01$; Figure 1B). By $24 \mathrm{~h}$, CrEDTA-ACRF1 and 2 had values of 85.8 and $90.8 \%$, respectively, of CrEDTA-water. This reflects an apparent dissociation of Cr from EDTA of 14.2 and $9.2 \%$, respectively. We term the absorbance change at peak wavelength "apparent dissociation" as it reflects the deviation of metal-EDTA preparations with ACRF from those in water or MES, but it does not differentiate absorbance contributions of the metal-EDTA from those of non-CrEDTA or non-CoEDTA compounds that were formed during the incubation with ACRF. Without knowledge of what compounds were evolved and their absorbance patterns across the wavelengths, it was not possible to correct for their effects. Consequently, the degree of metal-EDTA dissociation may have been greater than detected by spectrophotometry, but it is unlikely that it was less. The likelihood of $\mathrm{Cr}$ or Co reassociating with EDTA would seem small once the metal was associated with a compound for which it had greater affinity, unless that compound degraded.

The CoEDTA preparations in MES and in pH-adjusted ACRF differed in $\mathrm{pH}(P<0.01$; SED $=0.004$; Figure 1E). However, the differences were small, with maximum and minimum values for all samples of 6.05 and 6.00; the small differences were likely detected because the values were so consistent. There was no effect of incubation time on $\mathrm{pH}(P=0.37)$. Absorbance at $535 \mathrm{~nm}$ was consistent over time for CoEDTA-MES, but that in ACRF showed a steady decline over the incubation time (diluent $\times$ time, $P<0.01$; SED $=0.0005$; Figure $1 \mathrm{~F}$ ), with the $2 \mathrm{ACRF}$ preparations differing from each other $(P<0.01)$. By $24 \mathrm{~h}$, CoEDTA-MES was at $100.9 \%$ and +0.0015 absorbance units of its $0 \mathrm{~h}$ value, showing that it had maintained its absorbance throughout the incubation. In contrast, although the CoEDTA-ACRF preparations had absorbance values at $535 \mathrm{~nm}$ of 100.9 and $100.3 \%$ of the CoEDTA-MES at $0 \mathrm{~h}$, by $24 \mathrm{~h}$ they had declined to 96.3 and $95.7 \%$ of the CoEDTA-MES value, which give apparent dissociations of Co from EDTA of 3.7 and $4.3 \%$, respectively. The likelihood is low that changes in CoEDTA absor- bance in the present study were due to reduction of Co(III)EDTA to Co(II)EDTA and associated changes in peak absorbance wavelength from 535 to $465 \mathrm{~nm}$, respectively (Xue and Traina, 1996), because the ACRF preparations and incubations were carried out without effort to maintain anaerobicity and a reduced environment. Additionally, Co(II)EDTA has lower absorbances than Co(III)EDTA in the range of approximately 340 to $420 \mathrm{~nm}$ (Xue and Traina, 1996), a response that was not apparent for CoEDTA-ACRF in the present study.

Declines noted in absorbance of markers incubated in ACRF as compared with those in water or buffer suggest that the EDTA salts dissociated in ACRF. In preliminary studies using buffers containing $\mathrm{NaOH}$, $\mathrm{KCl}, \mathrm{KH}_{2} \mathrm{PO}_{4}$, and $\mathrm{KOH}$, absorbances at $541 \mathrm{~nm}$ of CrEDTA in solutions containing phosphorus were lower at 0 and $24 \mathrm{~h}$ than preparations made in water or buffer not containing phosphorus (data not shown). This would suggest that the lower values at $0 \mathrm{~h}$ seen with ACRF are not artifacts of using rumen fluid. The greater decline in absorbance of CrEDTA as compared with CoEDTA are in agreement with their stability constants as metals complexed with EDTA which are 23.4 for $\mathrm{Cr}$ (III) and 41.5 for Co(III) (Anderegg, 1977).

Although the CrEDTA-water and CoEDTA-MES showed little variation in absorbance at wavelengths of 380 and $330 \mathrm{~nm}$ over $24 \mathrm{~h}$, the markers in ACRF showed greater variability (Figures $1 \mathrm{C}, 1 \mathrm{D}, 1 \mathrm{G}$, and $1 \mathrm{H}$ ). At $380 \mathrm{~nm}$, absorbance values of CrEDTA preparations differed from each other over time (diluent $\times$ time, $P$ $<0.01$, SED $=0.0078$ ), with the water preparation giving a relatively flat line, and the ACRF preparations undergoing increases and decreases in values as the incubation progressed. The CrEDTA-water tended to differ from CrEDTA-ACRF $(P=0.06)$, but the 2 CrEDTA-ACRF did not differ from each other $(P=$ 0.19). The response of CrEDTA at $330 \mathrm{~nm}$ was similar in pattern to that at $380 \mathrm{~nm}$, with preparations differing over time (diluent $\times$ time, $P<0.01$, SED 0.0127; water vs. ACRF, $P=0.01$; ACRF1 vs. $2, P=0.23)$. The increases and decreases noted in absorbance in ACRF could reflect $\mathrm{Cr}$ going through a series of reactions that had differing effects on absorbance. The wavelength scan $\times$ time graphs of data from a CrEDTA analytical run show the degree to which the absorbances at wavelengths from 330 to $700 \mathrm{~nm}$ changed over time with CrEDTA-ACRF deviating from CrEDTA-water (Figures 2B and 2C).

As compared with CrEDTA, the lesser degree to which CoEDTA apparently dissociated in ACRF could be related to the less dramatic changes in absorbance for CoEDTA at shorter wavelengths over time. For CoEDTA at $380 \mathrm{~nm}$, there was a diluent $\times$ time effect $(P<0.01$, SED 0.0044), but with MES not differing 
CrEDTA
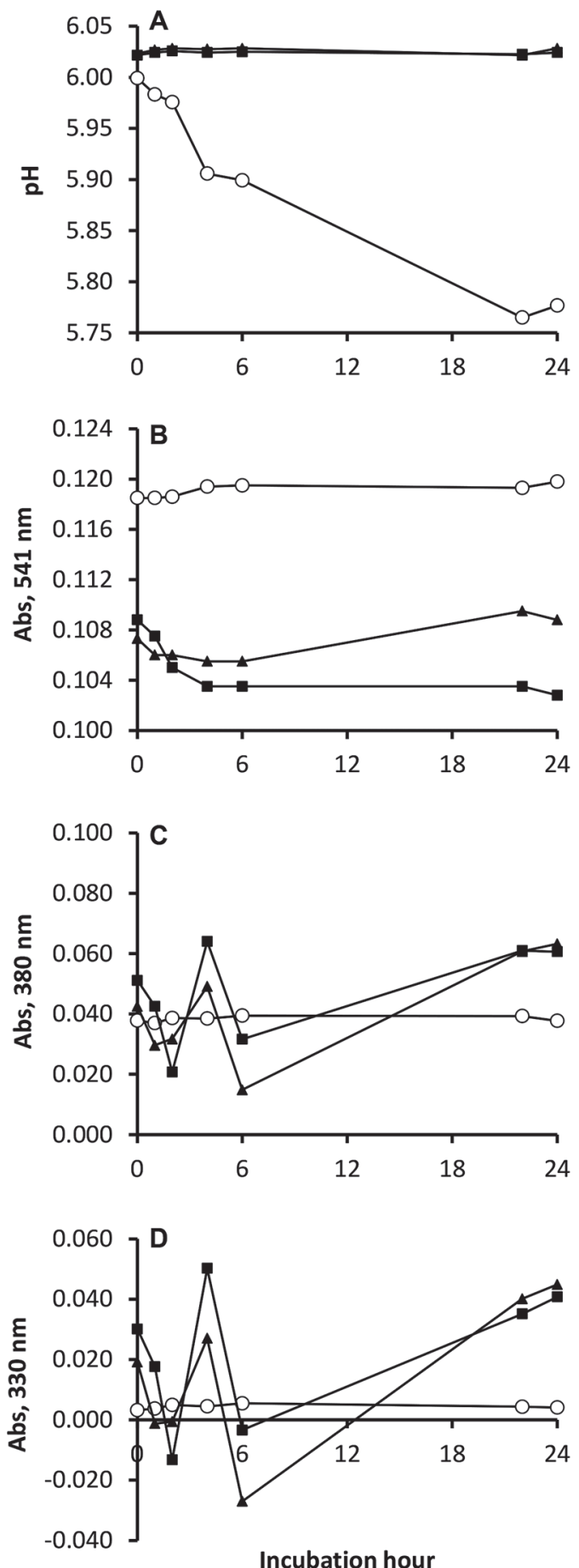

COEDTA
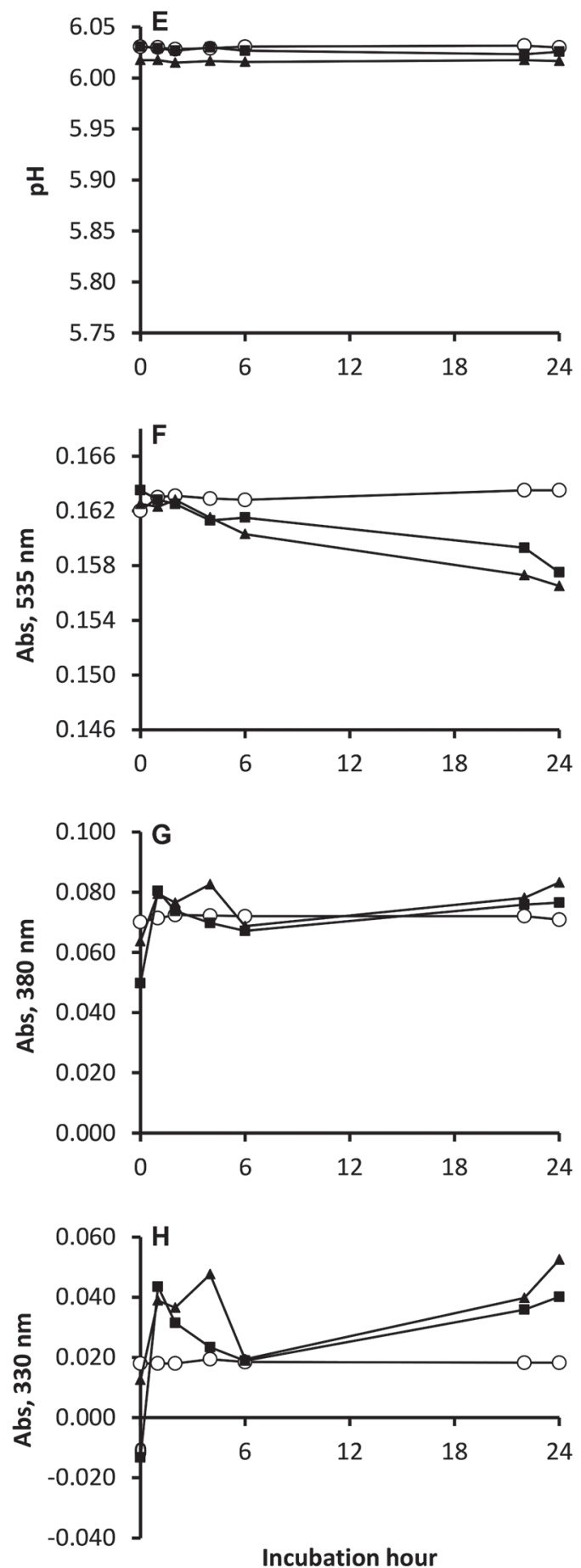

Figure 1. Absorbance (Abs) values over 24-h incubations of sample solutions for chromium-EDTA (CrEDTA) and cobalt-EDTA (CoEDTA) evaluations. Values are LSM. CrEDTA: $(\mathrm{A})=\mathrm{pH}$ [standard error of the difference $(\mathrm{SED})=0.024]$, $(\mathrm{B})=$ absorption at $541 \mathrm{~nm}(\mathrm{SED}=$ $0.0006),(\mathrm{C})=$ absorption at $380 \mathrm{~nm}(\mathrm{SED}=0.0078)$, and $(\mathrm{D})=$ absorption at $330 \mathrm{~nm}(\mathrm{SED}=0.0127) . \mathrm{CoEDTA}:(\mathrm{E})=\mathrm{pH}(\mathrm{SED}=0.004)$, $(\mathrm{F})=$ absorption at $535 \mathrm{~nm}(\mathrm{SED}=0.0005),(\mathrm{G})=$ absorption at $380 \mathrm{~nm}(\mathrm{SED}=0.0044)$, and $(\mathrm{H})=$ absorption at $330 \mathrm{~nm}(\mathrm{SED}=0.0078)$. Absorbances at 541 and $535 \mathrm{~nm}$ were obtained using cuvettes in a spectrophotometer, and at 330 and $380 \mathrm{~nm}$ in a plate reader. $\bigcirc=$ marker in water or MES buffer; $\mathbf{\square}=$ autoclaved clarified rumen fluid 1 or $3 ; \boldsymbol{\Lambda}=$ autoclaved clarified rumen fluid 2 or 4 . 
CrEDTA
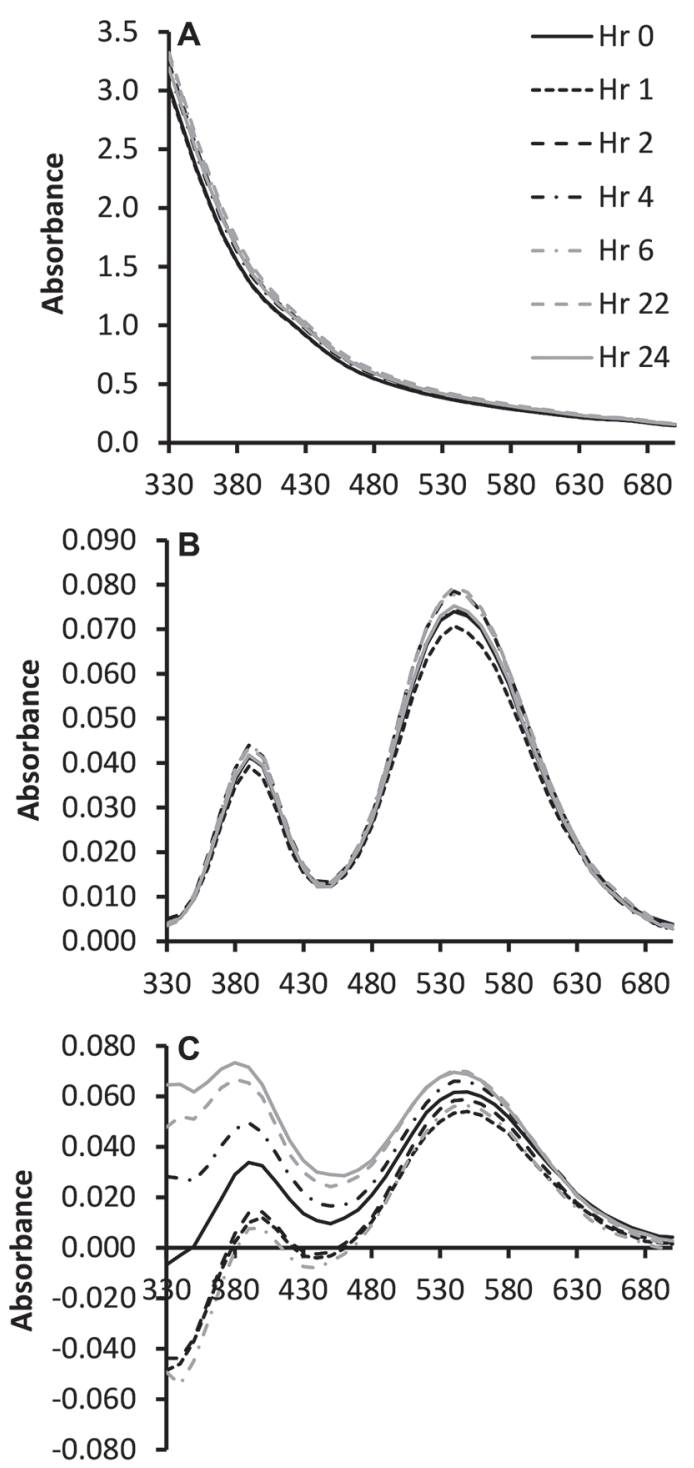

Wavelength
COEDTA
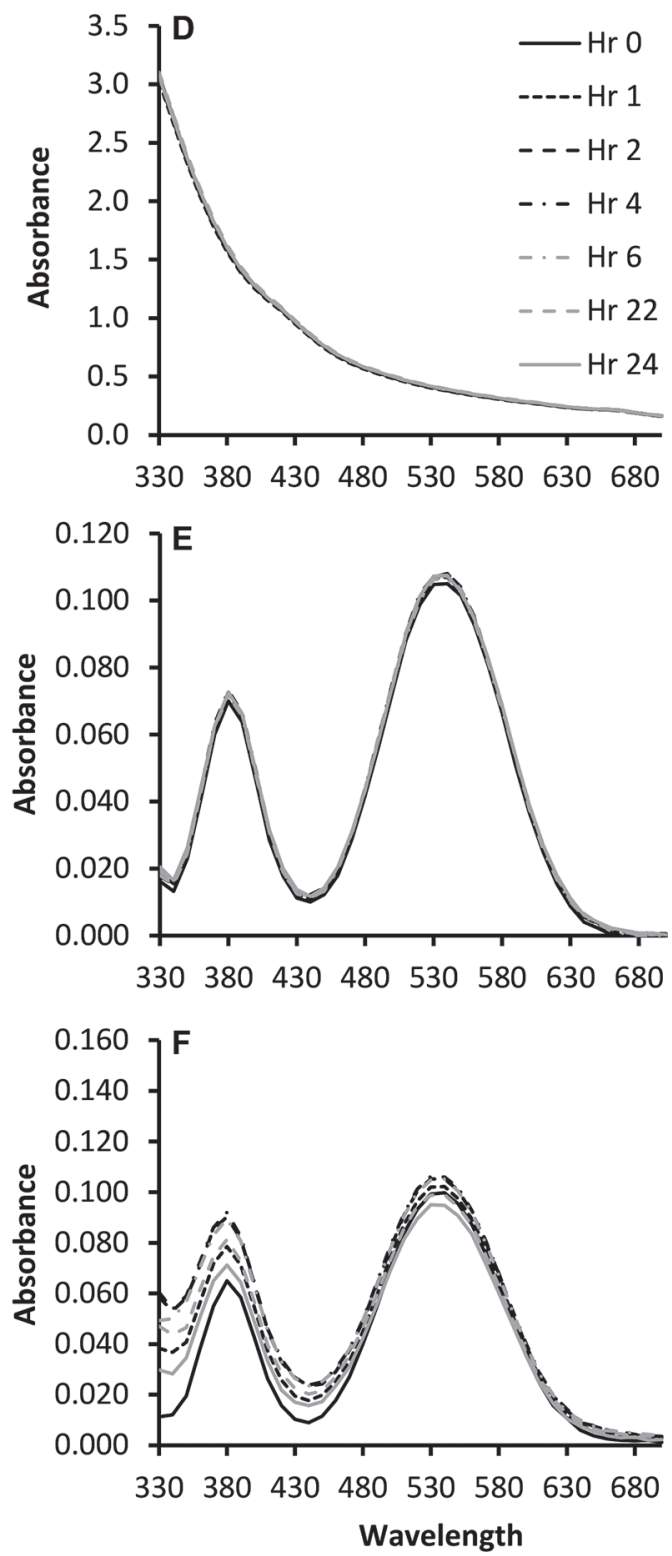

Figure 2. Wavescan absorbance data from 330 to $700 \mathrm{~nm}$ for chromium-EDTA (CrEDTA) run 9 and cobalt-EDTA (CoEDTA) run 12. Data are arithmetic averages of sample replicates within the analytical run measured in a plate reader. (A and D) Absorbance values for autoclaved clarified rumen fluid (ACRF) reagent blanks, (B) CrEDTA with water diluent, (C) CrEDTA with ACRF2 diluent, (E) CoEDTA with MES buffer diluent, and (F) CoEDTA with ACRF4 diluent. Lines show wavelength scans at individual sampling hours (Hr); legends as in panels A and D.

from $\operatorname{ACRF}(P=0.29)$ and the ACRF differing from each other $(P=0.01)$; the CoEDTA-ACRF appeared to experience less dramatic swings than did CrEDTAACRF. At $330 \mathrm{~nm}$, both ACRF3 and ACRF4 preparations showed greater changes in absorbance values over time than CoEDTA-MES (diluent $\times$ time $P<0.01$, SED 0.0078; MES vs. ACRF, $P<0.01$, ACRF3 vs. ACRF4, $P=0.02$ ). Visual comparison of the graphs of data from a CoEDTA analytical run suggest that the absorbances at 330 to $700 \mathrm{~nm}$ wavelengths over time for marker in ACRF appeared to deviate less from CoEDTA-MES (Figures 2E and 2F) than CrEDTAACRF differed from CrEDTA-water.

The changes in absorbance noted for markers in $\mathrm{ACRF}$ at 380 and $330 \mathrm{~nm}$ may be indicative of some of the species within ACRF with which markers were reacting. Phenolic compounds can bind metal cations including Co (Naumann et al., 2017) and $\mathrm{Cr}$ (Oo and 
Jain, 2007; Goswami et al., 2014) and are among the species that absorb in the 330 to $380 \mathrm{~nm}$ wavelength range (Anouar et al., 2012). Teeter and Owens (1983) reported that CrEDTA but not CoEDTA had an affinity for cottonseed hulls; in that study, they attributed the affinity and reduced recovery of PEG with cottonseed hulls as possibly due to a water-soluble compound such as tannins. In addition to the potentially rumen fluid-soluble phenolic compounds present in plant material, soluble lignin-carbohydrate complexes that may be released during digestion were found in cell free rumen liquor (Gaillard and Richards, 1975) and could be another source of phenolics that could react. That soluble phenolic compounds may be involved in the exchange of metals with EDTA does not preclude involvement of other anionic components in rumen fluid including phosphate and phytate.

\section{Potential Consequences of Marker Dissociation}

The significance of marker dissociation as it affects their suitability for research depends upon the fate of the marker components and whether they possess reactivity that alters digesta, measures of digesta passage, or animal metabolic responses. If the microbial or animal responses studied are to be applied to the larger population of animals that do not receive markers, nonreactivity of the markers is essential, unless it can be proved that the effects have no bearing on the response of interest. Released EDTA is not likely to degrade under anaerobic conditions (Tiedje, 1977) and could bind other minerals. If released $\mathrm{Co}$ and $\mathrm{Cr}$ react with phenolics or other ligands in the liquid fraction and remain tightly bound, the metals could still serve as nonreactive liquid markers; however, it is not known to what degree this occurs. If released metals were unreactive and continued to pass with the liquid digesta, their utility as markers could still be acceptable.

Evidence indicates that an apparently dissociated metal-EDTA marker can affect animal response. Cobalt-EDTA but not CrEDTA was shown to alter the profile of fatty acids in the milk of dairy cows (Shingfield et al., 2008), suggesting that CoEDTA dissociated and became reactive. In that study, CoEDTA was infused continuously into the rumen at $1.6 \mathrm{~g} / \mathrm{d}$ of Co for $7 \mathrm{~d}$ and the authors attributed changes in milk fatty acids to inhibition of $\Delta^{9}$-desaturase activity in the mammary gland. A study in Wistar rats showed that vitamin $\mathrm{B}_{12}$ deficiency relative to folic acid adequacy or excess alters activity of hepatic desaturases (Wadhwani et al., 2012); however, the basis for a vitamin $\mathrm{B}_{12}$ deficiency in the Shingfield et al. (2008) study is not clear. Although vitamin $\mathrm{B}_{12}$ was not measured in that study, Co as Co carbonate (S. Ahvenjärvi, Natu- ral Resources Institute, Jokioinen, Finland, personal communication) was added to the diets in amounts adequate to meet NRC (2001) requirements, suggesting that the animals were not likely to be vitamin $\mathrm{B}_{12}$ deficient. The CoEDTA infused in that study represented $727 \times$ the daily required amount of dietary Co (NRC, 2001). Two options for the observed effect of Co may be through increased production of vitamin $\mathrm{B}_{12}$ analogs, or as absorbed Co. An effectual $\mathrm{B}_{12}$ deficiency could be produced if the infused CoEDTA dissociated and greatly increased the ruminally available Co. In that situation, it is possible that formation of vitamin $\mathrm{B}_{12}$ analogs also increased (Kawashima et al., 1997) and inhibited activity of enzymes by binding in place of coenzymes derived from $\mathrm{B}_{12}$ but without their activity (Lengyel et al., 1960). Alternatively, Co as Co acetate has been shown to alter mammary desaturase activity in dairy cows. Oral $(3.5 \mathrm{~g}$ of $\mathrm{Co} / \mathrm{d})$ or intravenous (175 $\mathrm{mg}$ of $\mathrm{Co} / \mathrm{d}$ ) administration of Co acetate reduced milk fatty acid desaturation products in cows (Taugbøl et al., 2010); the oral dose of Co was approximately 1,500× of recommendations (NRC, 2001), and the venous dose was $5 \%$ of the oral dose to be comparable to urinary Co excretion noted with markers. Feed intake and milk yield were reduced with increasing ruminal infusion of Co in one study (Co acetate; Karlengen et al., 2013), but were unaffected in another (CoEDTA; Shingfield et al., 2008). The carryover effect on mammary fatty acid desaturation products appears to vary by dose level, and may be greater than $10 \mathrm{~d}$ (Karlengen et al., 2013). The degree to which CoEDTA dissociated in the present study seems inadequate to cause the effects seen in vivo with much higher doses of Co acetate. This suggests that there is an alternative mechanism for the effect of CoEDTA or for its dissociation other than association with compounds present in rumen fluid. The reduction of $\mathrm{Co}(\mathrm{III})$ to $\mathrm{Co}(\mathrm{II})$ associated with EDTA occurs at redox potentials of -60 to $-68 \mathrm{mV}$ (Ogino and Ogino, 1983), which are less negative than redox potentials achieved in the rumen $(-174$ to $-217 \mathrm{mV}$; Marden et al., 2005). It is possible that reduction in the rumen results in formation of $\mathrm{Co}$ (II)EDTA which has a substantially lower stability constant than $\mathrm{Co}$ (III) EDTA (16.5 and 41.5, respectively; Anderegg, 1977), and may be more prone to dissociation. This aspect of the markers remains to be investigated. Reports of effects of CrEDTA on animal or microbial metabolism or diet digestibility were not found in the literature.

\section{CONCLUSIONS}

Both CrEDTA and CoEDTA incubated in autoclaved clarified rumen fluid showed declines in absorbance over time at their maximum absorption wavelengths, sug- 
gesting that the metals dissociated from EDTA. The decline was greater for CrEDTA than for CoEDTA. Changes in the absorbance values at 330 and $380 \mathrm{~nm}$ suggest that phenolic compounds soluble in rumen fluid may be involved in the exchange, but that does not preclude involvement of other soluble species. The suitability of metal-EDTA markers for research will be dependent on the degree to which they dissociate, become reactive, and affect responses that affect treatment inferences that are intended to be applied to the larger population of animals that do not receive a marker.

\section{ACKNOWLEDGMENTS}

Special thanks go to J. W. Pitas of USDA-Agricultural Research Service US Dairy Forage Research Center, Madison, Wisconsin, for analytical assistance, and to the anonymous reviewers of this manuscript. This material is based upon work supported with funding from USDA-Agricultural Research Service. Mention of any trademark or proprietary product in this paper does not constitute a guarantee or warranty of the product by the USDA or the Agricultural Research Service and does not imply its approval to the exclusion of other products that also may be suitable.

\section{REFERENCES}

Anderegg, G. 1977. Critical survey of stability constants of EDTA complexes. IUPAC Chemical Data Series No. 14. Pergamon Press, Oxford, UK.

Anouar, E. H., J. Gierschner, J.-L. Duroux, and P. Trouillas. 2012. UV/visible spectra of natural polyphenols: A time dependent density functional theory study. Food Chem. 131:79-89.

AOAC International. 2005. Official Methods of Analysis, 18th ed. Association of Official Analytical Chemists, Washington, DC.

Binnerts, W. T., A. Th. van't Klooster, and A. M. Frens. 1968. Soluble chromium indicator measured by atomic absorption in digestion experiments. Vet. Rec. 82:470.

Cerar, J. 2015. Reaction between chromium (III) and EDTA ions: An overlooked mechanism of case study reaction of chemical kinetics. Acta Chim. Slov. 62:538-545.

Dobson, A., A. F. Sellers, and V. H. Gatewood. 1976. Dependence of Cr-EDTA absorption from the rumen on luminal osmotic pressure. Am. J. Physiol. 231:1595-1600.

Downes, A. M., and I. W. McDonald. 1964. The chromium-51 complex of ethylenediamine tetraacetic acid as a soluble rumen marker. Br. J. Nutr. 18:153-162.

Faichney, G. J. 1993. Digesta flow. Pages 53-85 in Quantitative Aspects of Ruminant Digestion and Metabolism. J. M. Forbes and J. France, ed. CAB International, Wallingford, UK.

Gaillard, B. D. E., and G. N. Richards. 1975. Presence of soluble lignin-carbohydrate complexes in the bovine rumen. Carbohydr. Res. 42:135-145.

Goswami, S., A. K. Das, A. K. Maity, A. Manna, K. Aich, S. Maity, P. Saha, and T. K. Mandal. 2014. Visual and near IR (NIR) fluorescence detection of $\mathrm{Cr}^{3+}$ in aqueous media via spirobenzopyran ring opening with application in logic gate and bio-imaging. Dalton Trans. 43:231-239.

Hall, M. B., and P. J. Van Soest. 2019. Liquid digesta markers: A method for synthesis of crystallized chromium-EDTA and com- parison of its degree of complexation with an uncrystallized preparation. Anim. Feed Sci. Technol. 253:32-38.

Hamm, R. E. 1953. Complex ions of chromium. IV. The ethylenediaminetetraacetic acid complex with chromium (III). J. Am. Chem. Soc. 75:5670-5672.

Hedrick, C. E. 1965. Formation of the chromium-EDTA complex. J. Chem. Educ. 42:479-480.

Karlengen, I. J., O. Taugbøl, B. Salbu, A. H. Aastveit, and O. M. Harstad. 2013. Effect of different levels of supplied cobalt on the fatty acid composition of bovine milk. Br. J. Nutr. 109:834-843.

Kawashima, T., P. R. Henry, D. G. Bates, C. B. Ammerman, R. C. Littell, and J. Price. 1997. Bioavailability of cobalt sources for ruminants. 3 . In vitro ruminal production of vitamin $\mathrm{B}_{12}$ and total corrinoids in response to different cobalt sources and concentrations. Nutr. Res. 17:975-987.

Lengyel, P., R. Mazumder, and S. Ochoa. 1960. Mammalian methylmalonyl isomerase and vitamin B12 coenzymes. Proc. Natl. Acad. Sci. USA 46:1312-1318.

Marden, J. P., C. Bayourthe, F. Enjalbert, and R. Moncoulon. 2005. A new device for measuring kinetics of ruminal $\mathrm{pH}$ and redox potential in dairy cattle. J. Dairy Sci. 88:277-281.

Naumann, H. D., L. O. Tedeschi, W. E. Zeller, and N. F. Huntley. 2017. Invited review. The role of condensed tannins in ruminant animal production: Advances, limitations and future directions. Rev. Bras. Zootec. 46:929-949.

NRC. 2001. Nutrient Requirements of Dairy Cattle. 7th rev. ed. National Academy Press, Washington, DC.

Ogino, H., and K. Ogino. 1983. Redox potentials and related parameters of cobalt (III/II) complexes containing aminopolycarboxylates. Inorg. Chem. 22:2208-2211.

Oo, C. W., and K. Jain. 2007. Chromium (III) adsorption from aqueous solution by Rhizophora apiculata tannins. Indo. J. Chem. $7: 180-184$

Paraneeiswaran, A., S. K. Shukla, V. S. Sathyaseelan, and T. S. Rao. 2015. A spectrophotometric method for the determination Co-EDTA complexes. Int. J. Appl. Sci. Biotechnol. 3:584-587.

Shingfield, K. J., A. Arölä, S. Ahvenjärvi, A. Vanhatalo, V. Toivonen, J. M. Griinari, and P. Huhtanen. 2008. Ruminal infusions of cobalt-EDTA reduce mammary $\Delta 9$-desaturase index and alter milk fatty acid composition in lactating cows. J. Nutr. 138:710-717.

Taugbøl, O., I. J. Karlengen, B. Salbu, A. H. Aastveit, and O. M. Harstad. 2010. Intravenous injections of cobalt reduce fatty acid desaturation products in milk and blood of lactating cows. J. Anim. Physiol. Anim. Nutr. (Berl.) 94:635-640.

Teeter, R. G., and F. N. Owens. 1983. Characteristics of water soluble makers for measuring rumen liquid volume and dilution rate. J. Anim. Sci. 56:717-728.

Tiedje, J. M. 1977. Influence of environmental parameters on EDTA biodegradation in soils and sediments. J. Environ. Qual. 6:21-26.

Udén, P., P. E. Colucci, and P. J. Van Soest. 1980. Investigation of chromium, cerium and cobalt as markers in digesta. Rate of passage studies. J. Sci. Food Agric. 31:625-632.

Wadhwani, N. S., R. R. Manglekar, K. D. Dangat, A. V. Kulkarni, and S. R. Joshi. 2012. Effect of micronutrients (folic acid, vitamin $\left.\mathrm{B}_{12}\right)$ and omega 3 fatty acids on liver fatty acid desaturases and transport proteins in Wistar rats. Prostaglandins Leukot. Essent. Fatty Acids 86:21-27.

Weimer, P. J., Y. Shi, and C. L. Odt. 1991. A segmented gas-liquid delivery system for continuous culture of microorganisms on insoluble substrates and its use for growth of Ruminococcus flavefaciens on cellulose. Appl. Microbiol. Biotechnol. 36:178-183.

Weisbjerg, M. R., T. Hvelplund, and B. M. Bibby. 1998. Hydrolysis and fermentation rate of glucose, sucrose and lactose in the rumen. Acta Agric. Scand. A Anim. Sci. 48:12-18.

Xue, Y., and S. J. Traina. 1996. Oxidation kinetics of Co(II)-EDTA in aqueous and semi-aqueous goethite suspensions. Environ. Sci. Technol. 30:1975-1981. 